\title{
Explanation of Translation and Citation
}

The book has been translated by Chadwick Smith (New York).

In some cases his translation is based on a previous lecture version of the chapter done by other translators: Georgina Paul, Chapters I, 2, and 4; and Jeremy Gaines, Chapters 3, 7, and 9.

Since a great number of the citations from Walter Benjamin's writings have been modified (for reasons that are explained in detail in several footnotes and at length in Chapter 7), the book would become unreadable if this were explicitly indicated in every case. The manner of citation is therefore indicated within the parenthetical references as follows:

- If only the German source is indicated, it is our own translation.

- Where only the standard English translation is indicated, we follow these translations.

- In cases where both versions are indicated (the German source preceding the English publication), this formatting indicates translation modified. 
